\title{
Altered patterns of cortical activation in ALS patients during attention and cognitive response inhibition tasks
}

\author{
L. H. Goldstein - I. C. Newsom-Davis • \\ V. Bryant $\cdot$ M. Brammer $\cdot$ P. N. Leigh $\cdot$ \\ A. Simmons
}

Received: 17 January 2011/ Accepted: 28 April 2011/Published online: 10 May 2011

(C) The Author(s) 2011. This article is published with open access at Springerlink.com

\begin{abstract}
Since amyotrophic lateral sclerosis (ALS) can be accompanied by executive dysfunction, it is hypothesised that ALS patients will have impaired performance on tests of cognitive inhibition. We predicted that ALS patients would show patterns of abnormal activation in extramotor regions when performing tests requiring the inhibition of prepotent responses (the Stroop effect) and the inhibition of prior negatively primed responses (the negative priming effect) when compared to healthy controls. Functional magnetic resonance imaging was used to measure activation during a sparse sequence block design paradigm investigating the Stroop and negative priming effects in 14 ALS patients and 8 healthy age- and IQmatched controls. Behavioural measures of performance were collected. Both groups' reaction times (RTs) reflected the Stroop effect during scanning. The ALS and control groups did not differ significantly for any of the behavioural measures but did show significant differences in
\end{abstract}

L. H. Goldstein $(\varangle) \cdot$ I. C. Newsom-Davis · V. Bryant

King's College London, Department of Psychology, Institute of Psychiatry, MRC Centre for Neurodegeneration Research, PO77, De Crespigny Park, London SE5 8AF, UK e-mail: laura.goldstein@kcl.ac.uk

M. Brammer · A. Simmons

King's College London, Institute of Psychiatry,

Centre for Neuroimaging Sciences, London, UK

P. N. Leigh

King's College London, Department of Clinical Neuroscience, Institute of Psychiatry, MRC Centre for Neurodegeneration

Research, London, UK

P. N. Leigh · A. Simmons

NIHR Biomedical Research Centre for Mental Health

at South London and Maudsley NHS Foundation Trust

and King's College London, Institute of Psychiatry, London, UK cerebral activation during both tasks. The ALS group showed increased activation predominantly in the left middle temporal gyrus (BA 20/21), left superior temporal gyrus (BA 22) and left anterior cingulate gyrus (BA 32). Neither group's RT data showed clear evidence of a negative priming effect. However the ALS group showed decreased activation, relative to controls, particularly in the left cingulate gyrus (BA 23/24), left precentral gyrus (BA 4/6) and left medial frontal gyrus (BA 6). Greater cerebral activation in the ALS group accompanying the performance of the Stroop effect and areas of decreased activation during the negative priming comparison suggest altered inhibitory processing in ALS, consistent with other evidence of executive dysfunction in ALS. The current findings require further exploration in a larger study.

Keywords Amyotrophic lateral sclerosis · fMRI · Cognitive function $\cdot$ Response inhibition

\section{Introduction}

Depending on the criteria used, $35-50 \%$ of people with amyotrophic lateral sclerosis (ALS) may show cognitive impairment, particularly executive dysfunction (e.g. [1-4]) with milder deficits of memory and language (e.g. [5]). The most widely reported deficits have been on tests of verbal fluency where a formal adjustment has been made for motor or speech impairments (e.g. [6-10]) as well as in other studies [11-13]. Neuroimaging studies have related verbal fluency impairment to altered patterns of cerebral activation $[8,11,14-16]$ and to the reduction of white matter volume in frontotemporal association fibres [17].

Less consideration has been given to the cerebral activation underlying cognitive function in ALS using other 
cognitive, extramotor paradigms [18], particularly assessing attention. Although on the basis of a meta-analysis it was concluded that ALS patients do not show attentional impairments [2], deficits have been reported on tests of visual attention [19]. The Stroop test, which measures focused attention and response inhibition, has yielded contradictory results [11, 20-23]. In the Stroop test [24], there is an increase in response time when naming the colour of the ink in which an incongruous colour name is displayed (e.g. when the participant has to say 'blue' in response to the word 'RED' printed in blue ink) in comparison to control conditions where the stimulus characteristics do not conflict with each other (e.g. RED printed in red ink). This is known as the Stroop effect. In a previous study [6] no difference in the Stroop effect was found when comparing the performance of 52 ALS patients and healthy controls. However, a strong trend $(p=0.051)$ for a between-group difference was found on a further adaptation of the Stroop test, known as negative priming (NP) [25]. In the NP task, participants are also required to name the colour of the ink in which an incongruous colour word is printed, but the colour of the ink is the same as the colour word on the previous trial, e.g. RED in blue ink is followed by GREEN in red ink. Thus the correct response on any trial is the stimulus that on the previous trial had to be ignored as a distractor. The task, therefore, requires the person to inhibit responses on a particular trial and then make the previously inhibited response on the next trial. In healthy controls, the NP task produces increased (i.e. slower) response times compared to the Stroop effect. The NP task has therefore been interpreted as making greater demands on active inhibitory cognitive processes [26]. Two theoretical models have been used to account for the NP effect, the selective inhibition account and the episodic retrieval model [27]. In our previous study [6], the ALS group showed shorter response latencies compared to controls for the NP effect. This was interpreted as suggesting impairment of normal cognitive inhibitory processes. In this scenario, less cognitive inhibition of a stimulus on trial ' $N$ ' would make this stimulus easier to respond to on trial ' $N+1$ ' and would lead to a shorter response time than would a stronger level of cognitive inhibition on trial ' $N$ '. In addition, if there is impaired cognitive inhibition (which in the Stroop task is reflected by longer response times resulting from poorer inhibition of the colour word when naming the colour ink is required), this would 'help' the ALS patients in the NP trials and lead to a reduced NP effect. Elsewhere, patients with frontal lobe damage have also been reported to show reduced NP effects [28], which further suggests altered cognitive inhibitory processes. Other clinical groups thought to demonstrate reductions in cognitive inhibition have also shown a reduced NP effect [29, 30].
In healthy controls, fMRI has demonstrated predominantly the involvement of the cingulate gyrus, the inferior, superior and medial frontal gyrus and the hippocampal gyrus during the Stroop test $[26,31,32]$. The inferior, superior and middle frontal gyrus, superior and middle temporal as well as inferior parietal lobe and thalamus were among the regions found to show differential activation comparing performance during the NP test and the Stroop test [26]. Since a number of functional neuroimaging studies and neuropathology investigations have shown involvement of a selection of these regions in ALS (e.g. [8, $11,14,33,34])$, the present study set out to determine, in a pilot study, whether performance on Stroop and negative priming paradigms during fMRI scanning would elicit patterns of abnormal activation in extramotor regions known to be implicated more generally in cognitive impairment in ALS [4]. The study also set out to explore whether the patterns of cerebral activation in patients with ALS might elucidate the nature of inhibitory process changes seen in ALS in our previous study [6].

\section{Methods}

\section{Participants}

Fourteen people with possible, probable or definite ALS according to the revised El-Escorial criteria [35] were recruited from the King's MND Care and Research Centre in London. None had a clinical diagnosis of dementia. Individuals with a family history of ALS were excluded, as were people with very slow/unintelligible speech (which would make measuring performance on the Stroop/negative priming test impossible) or forced vital capacity $<80 \%$ as hypoventilation may impair cognitive function in ALS $[36,37]$. Eight control participants were recruited from among friends/spouses of the people with ALS or from among a departmental participant pool of healthy adults. Exclusion criteria for both patients and controls included: (1) not being right-handed; (2) a history of diabetes, stroke, head injury or loss of consciousness; (3) a history of a psychiatric or psychological disorder or currently taking psychotropic medication; (d) colour-blindness. More people with ALS than controls were recruited to incorporate the likely heterogeneity in cognitive ability seen in the disorder [1-4].

\section{Measures}

Symptom severity was measured using the ALS Severity Scale [38]. This scale, used in other scanning and neuropsychology studies by our group (e.g. [6-10, 14]) provides summary scores of bulbar and spinal function as well as a 
total score. Bulbar and spinal scores each have a maximum of 20 with higher scores indicating less functional impairment. Premorbid general intellectual ability was assessed using the National Adult Reading Test [39]. Anxiety and depression were measured using the Hospital Anxiety and Depression Scale (HADS) [40]. For each of the anxiety and depression subscales, scores between 0-7 are considered normal, $8-10$ represents a borderline state of anxiety or depression, and scores above 10 indicate "caseness". Depression scores with and without the item "I feel as if I am slowed down" [6, 8, 14] were calculated.

Age and clinical measures were compared between the ALS and healthy groups with the Mann-Whitney $U$ test, while gender was compared with Fisher's exact test.

The project received ethical approval from the Ethics Committee at the Institute of Psychiatry, King's College London. All participants gave written informed consent prior to participating in the study, which was performed in accordance with the ethical standards laid down in the 1964 Declaration of Helsinki.

\section{Neuroimaging paradigm}

\section{Control, Stroop and negative priming tasks}

Three sets of stimuli were presented to participants in the MRI scanner. Two sets of stimuli consisted of words (BLUE, GREEN, RED AND YELLOW) presented in another one of those colours, as well as control stimuli, which were strings of between three and six coloured crosses, presented in the colours above. In the Stroop condition, the incongruous colour of the ink in which the word was printed was unrelated to the word presented on the previous trial. For the NP stimuli, the colour ink was the same as the colour word on the previous trial (e.g. RED written in green ink was followed by BLUE written in red ink). In all conditions the participants were instructed simply to name the colour of the ink in which the stimuli were presented. No further information was given about how the colour ink and nature of the stimulus were related.

Three sets of stimuli were compiled in order to compare the Stroop versus control conditions, NP versus control conditions and Stroop versus NP. Within each set, there were five alternating blocks of trials per condition, with 8 trials per block. For all stimulus classes, participants were presented with a fixation cross for $1,800 \mathrm{~ms}$, the stimulus was presented for $100 \mathrm{~ms}$, and there was then a blank period (interstimulus interval; ISI) of $2.6 \mathrm{~s}$ during which the response was to be made. The response time was established on the basis of prior pilot work to provide sufficient response time for the ALS patients. Since the response to NP trials depends on the previous stimulus, the first trial was discarded; consequently in the calculation of results for all conditions the first trial of each block was discarded.

For each main set of trials (Control vs. Stroop; Control vs. NP; Stroop vs. NP) two different randomised trial presentations were generated, and the use of these two orders was counterbalanced across participants, as was the order in which the different blocks was presented. Voice-activated hardware recorded the reaction time (RT) to each stimulus, using a microphone headset to detect verbal responses. A button-box activated by the investigator was used to record the response made (i.e. the colour name). This was designed to allow the collection of performance data during the scan, which had not been a feature of earlier studies [26]. The number of correct and incorrect responses in each condition and the mean RT for each condition were automatically calculated. For each of the sets of trials, two average RTs were computed. The Stroop effect was calculated as Stroop RT minus Control RT; the NP effect was the NP RT minus the Stroop RT. Calculation of the Stroop effect controls for individual differences in speed of speech production, important in ALS, and therefore represents a relatively pure index of the degree of Stroop interference experienced (i.e. the difficulty in inhibiting word reading so that the ink colour can be named). The NP effect calculation also controls for speed of speech production but, additionally, controls for the passive cognitive inhibitory processes involved in Stroop interference so that the NP effect then represents a purer measure of the more active cognitive inhibitory processes thought to be involved in the inhibition of a previously primed stimulus. As a further means of controlling for motor speech differences or more general slowing, the Stroop RTs were also expressed as a percentage of the Control RTs, and the NP RTs were similarly expressed as a percentage of the Stroop RT values.

\section{Image acquisition}

Functional MRI data were acquired on a GE Signa NV/i 1.5-T MRI scanner (General Electric, Milwaukee, WI). One hundred T2*-weighted EPI volumes depicting BOLD (blood oxygenation level dependent) contrast were acquired in each of 16 non-contiguous near-axial planes parallel to the anterior commissure-posterior commissure line $(64 \times 64$ matrix, slice thickness $=7 \mathrm{~mm}$, slice gap $=0.7 \mathrm{~mm}, 3 \times 3 \times$ $7 \mathrm{~mm}$ voxel size, $\mathrm{TE}=40 \mathrm{~ms}, \mathrm{TR}=4,000 \mathrm{~ms}$, flip angle $=90^{\circ}$, number of signal averages $=1$ ). A sparse pulse sequence design was used; $1,400 \mathrm{~ms}$ of image acquisition was followed by $2,600 \mathrm{~ms}$ of quietness during which no images were acquired and the participant could speak the name of the colour aloud without any scanner noise. During the same session, a 43-slice, high-resolution inversion recovery echo planar image of the whole brain was acquired in the AC-PC plane $(128 \times 128$ matrix, slice thickness $=3 \mathrm{~mm}$, slice 
gap $=0.3 \mathrm{~mm}$, voxel size $=1.5 \times 1.5 \times 3 \mathrm{~mm}, \quad \mathrm{TE}=$ $73 \mathrm{~ms}, \mathrm{TR}=16,000 \mathrm{~ms}, \mathrm{TI}=180 \mathrm{~ms}$ ). Data acquisition from patients and controls was interleaved to ensure that a shift in scanner performance would not lead to spurious results. Echo planar imaging data quality was assessed using an automated analysis technique [41].

T2-weighted fast spin echo images were also acquired for each participant from 60 contiguous 3 -mm slices $(256 \times 256$ matrix, slice thickness $=3 \mathrm{~mm}$, slice gap $=0 \mathrm{~mm}$, voxel size $=0.9 \times 0.9 \times 3 \mathrm{~mm}, \mathrm{TE}=80 \mathrm{~ms}, \mathrm{TR}=3,000 \mathrm{~ms}$, echo train length $=8$ ) and were reviewed by an experienced neuroradiologist to exclude any participants demonstrating abnormalities unrelated to ALS.

\section{Image analysis}

All fMRI data analysis was undertaken using locally written software, which has been extensively validated [42-45]. All of the analysis up to the group stage was made blinded to the participant's identity.

Prior to time-series analysis, data were corrected for the effects of head motion in 3D as previously described [42]. The data were analysed using a general linear model in which the task design was convolved with a mixture of two one-parameter gamma variate functions (peak responses at 4 and $8 \mathrm{~s}$ ) to account for haemodynamic delay and dispersion. The time series at each voxel was regressed on the convolved design. The parameters obtained from the regression were used to calculate a "goodness of fit" statistic [the ratio of the sum of squares of the model fit and the residual sum of squares (SSQ ratio)] at every voxel. The significance of the statistic was then assessed by a data-driven permutation approach [43, 44].

Data for each individual were transformed into the standard space of Talairach and Tournoux [45, 46]. Differences in activation between groups were tested for significance using an analysis of variance (ANOVA) at each voxel in standard space [45]. The median value of the goodness of fit statistic was calculated at every voxel in standard space (medians were used to minimise outlier effects). The significance of these median values was then assessed using non-parametric, data-driven, non-permutation-based procedures[45] that were extended to the cluster level [47] in order to produce group difference images.

\section{Results}

\section{Participant characteristics}

The demographic and clinical data for the participants are presented in Table 1. This shows that the two groups were well matched for age, gender, estimates of premorbid Full
Scale IQ and mood. Although there was a trend for the ALS group to appear more depressed, once the item "I feel as if I am slowed down", which may reflect disease-related symptoms rather than depression, was removed from the calculation, there was no between-group difference on the HADS depression subscale. None of the participants were found to have any abnormalities on the T2-weighted structural images incompatible with a diagnosis of ALS.

\section{Behavioural performance during the fMRI tasks}

Table 2 illustrates the reaction time (RT) and error data for the ALS and control participants for all conditions. Due to technical failure, RTs and error responses could not be recorded across all conditions for two of the ALS participants. The RTs for the ALS group for the individual Control, Stroop and NP conditions were significantly longer than for the controls, as expected for ALS patients. These differences in motor aspects of responding were controlled for in the calculation of the Stroop and NP effects. Both groups showed RT evidence of a Stroop effect (i.e. Stroop RT minus Control RT; Wilcoxon signed ranks, Control group: $Z=-2.24, p=0.025$; ALS group: $Z=$ $-3.059, p=0.002$ ). However, neither group showed clear behavioural evidence of the NP effect in terms of differential RTs (i.e. NP RT minus Stroop RT; Wilcoxon signed ranks, Control group: $Z=-1.332, p=0.183$; ALS group: $Z=-1.412, p=0.158)$, although for both groups the mean NP RTs were the longest of the three mean RT values. There were no between-group differences on either the mean Stroop RT expressed as a percentage of the Control RT or the NP RT values expressed as a percentage of the Stroop RT values (Table 2). Although the betweengroup difference for the Stroop effect did not reach statistical significance at $p<0.05$ using non-parametric analyses (Table 2), given the small sample sizes the difference between the groups' Stroop effect was further considered by examining the effect size [48] for the between-group comparison. This permitted an estimation of the standardised mean difference between the two groups (i.e. the effect size). Standardised against the entire sample's RTs for the Control condition, the between-group difference for the Stroop effect reflected a large effect size of $d=0.809$ [48]. This suggested that there was greater interference in responding for the ALS group, but that the lack of statistical significance was likely to be due to the small sample size in the current study. The between-group difference for the NP effect was also not significant, although the NP for the ALS group appeared somewhat shorter than for controls. The between-group difference, standardised against the standard deviation of the entire sample's Stroop RTs, reflected a small effect size $(d=0.183)$ so that the difference in RTs will again not 
Table 1 Demographic and clinical characteristics of participants

\begin{tabular}{|c|c|c|c|}
\hline & ALS & Controls & Statistic \\
\hline Age (years) & $52.6(11.6)$ & $52.4(7.2)$ & Mann-Whitney $U=52.5, Z=-0.239, p=0.811$ \\
\hline Gender & $9 \mathrm{M}: 5 \mathrm{~F}$ & $6 \mathrm{M}: 2 \mathrm{~F}$ & Fisher's exact test $p=1.0$ \\
\hline NART-estimated IQ & $118.0(12.6)$ & $124.6(2.7)$ & $\begin{array}{l}\text { Mann-Whitney, } U=38.0 \\
Z=-1.023, p=0.306\end{array}$ \\
\hline HADS anxiety $(\max 21)$ & $7.1(5.1)$ & $5.8(2.3)$ & $\begin{array}{l}\text { Mann-Whitney } U=47.0 \\
Z=0.620, p=0.536\end{array}$ \\
\hline HADS depression $(\max 21)$ & $4.7(3.1)$ & $2.5(2.9)$ & $\begin{array}{l}\text { Mann-Whitney } U=31.5 \\
Z=-1.685, p=0.092\end{array}$ \\
\hline HADS depression (modified)* $(\max 18)$ & $2.7(2.7)$ & $1.8(2.0)$ & $\begin{array}{l}\text { Mann-Whitney } U=43.0 \\
Z=-0.909, p=0.363\end{array}$ \\
\hline ALSSS & & N/A & N/A \\
\hline Bulbar (max 20) & 16.5 (4.2) Range 8-20 & & \\
\hline Spinal (max 20) & 14.9 (3.9) Range 8-19 & & \\
\hline Total $(\max 40)$ & 32.1 (4.9) Range 23-39 & & \\
\hline
\end{tabular}

All values mean $(\mathrm{SD})$

NART National Adult Reading Test, HADS Hospital Anxiety and Depression Scale, ALSSS ALS Severity Scale

* Item "I feel as if I am slowed down" removed

have reached significance given the sample size. Although the two groups did not differ in the number of errors made during Control or Stroop trials, there was a trend for the ALS group to make more response errors during the NP trials than the healthy control group (Table 2).

fMRI analyses

\section{Stroop effect (Stroop minus control condition)}

Brain regions differentially activated in the ALS and healthy control groups during the Stroop minus control conditions are shown in Fig. 1, with details of the regions presented in Table 3.
All brain regions demonstrating significant betweengroup activation differences showed increased activation in the ALS group relative to controls. These regions were located almost entirely in the left hemisphere. The largest clusters were located in the left middle temporal gyrus (predominantly BA 20/21), left superior temporal gyrus (predominantly BA 22) and left anterior cingulate gyrus (predominantly BA 32). In addition, significant clusters were observed in the left fusiform and lingual gyri (both BA 18), left medial frontal gyrus (predominantly BA 9) and left inferior parietal cortex (BA 39/40). Subcortical activation differences were found in the hippocampus, caudate nucleus and insula, all on the left, as well as the cerebellum.

Table 2 Reaction times and error scores for scanning conditions and the overall Stroop and negative priming effects

\begin{tabular}{lccl}
\hline & ALS & Controls & Statistic \\
\hline Control condition RT (ms) & $802(190)$ & $641(149)$ & Mann-Whitney $U=18.5, Z=-2.277, p=0.023$ \\
Stroop condition RT(ms) & $932(207)$ & $717(146)$ & Mann-Whitney $U=13.0, Z=-2.70 p=0.007$ \\
Negative priming condition RT (ms) & $951(179)$ & $747(134)$ & Mann-Whitney $U=10.0, Z=-2.932, p=0.003$ \\
Stroop effect RT (ms) & $129(60)$ & $76(65)$ & Mann-Whitney $U=27.0, Z=-1.62 p=0.105$ \\
Negative priming effect RT (ms) & $20(53)$ & $29(53)$ & Mann-Whitney $U=46.0, Z=-0.154 p=0.877$ \\
Stroop RT expressed as percentage of control RT & $117(8)$ & $113(12)$ & Mann-Whitney $U=40.0, Z=-0.617, p=0.537$ \\
NP RT expressed as percentage of Stroop RT & $105(9)$ & $103(6)$ & Mann-Whitney $U=45.0, Z=-0.231, p=0.817$ \\
Errors during control condition & $0.4(1.3)$ & $0(0)$ & Mann-Whitney $U=44.0, Z=-0.816 p=0.414$ \\
Errors during Stroop condition & $0.3(0.6)$ & $0.1(0.2)$ & Mann-Whitney $U=41.5, Z=-0.720 p=0.472$ \\
Errors during negative priming condition & $0.6(1.6)$ & $0(0)$ & Mann-Whitney $U=32.0, Z=-1.767 p=0.077$ \\
\hline Alv & & &
\end{tabular}

All values mean $(\mathrm{SD})$ 


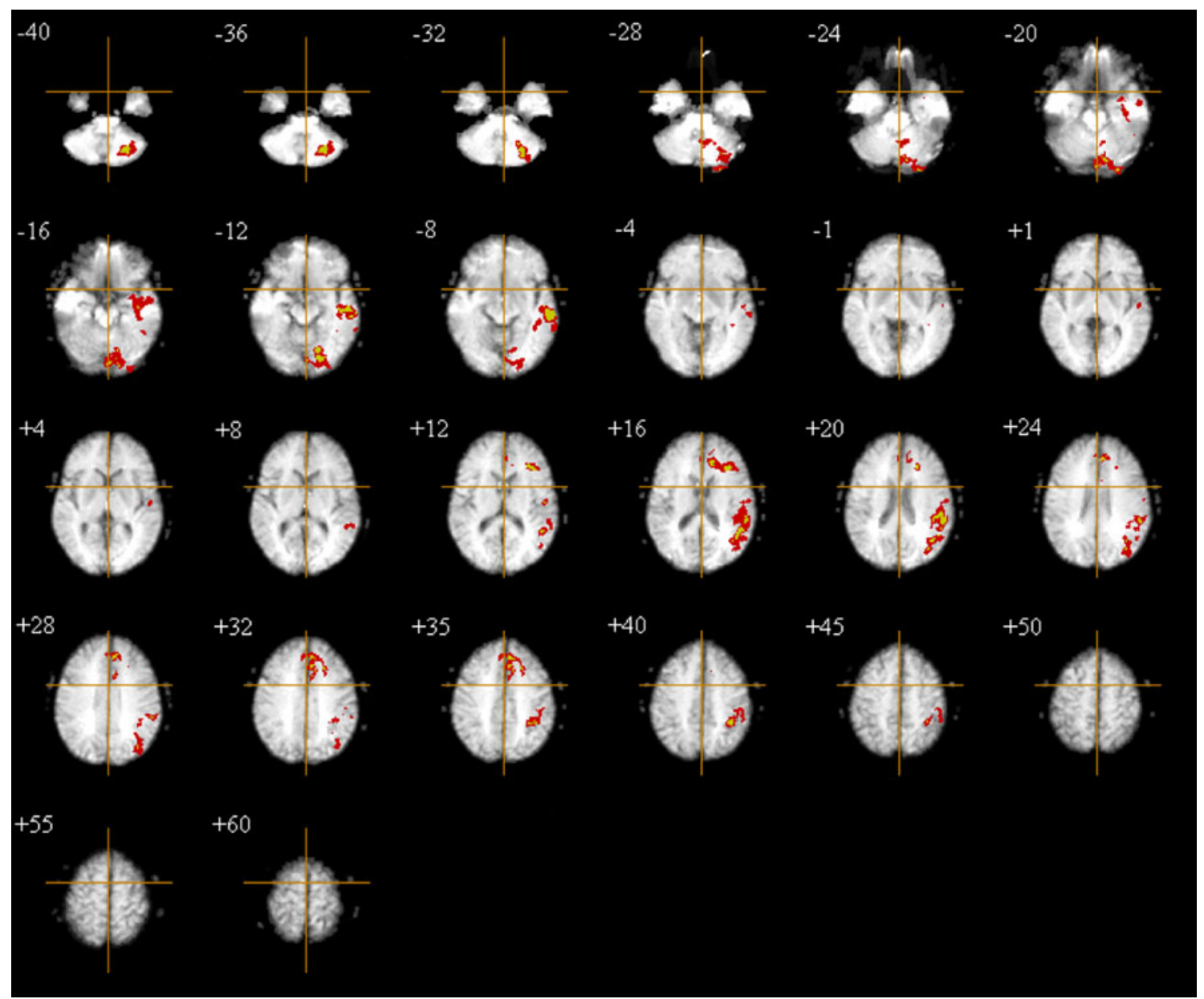

Fig. 1 Brain regions differentially activated in the ALS and healthy control groups during the Stroop minus Control conditions (i.e. the Stroop effect) at $p=0.001$. All regions demonstrating significant between-group activation differences (shown in red) showed

\section{Negative priming effect (NP minus Stroop condition)}

Brain regions differentially activated in the ALS and healthy control groups during the NP minus Stroop conditions are shown in Fig. 2, with details of the regions presented in Table 4.

For this comparison, all regions showing significant difference indicated reduced activation in the ALS group compared to controls; these regions were predominantly in the left hemisphere.

The largest cluster of reduced activation in the ALS group was located in the left cingulate gyrus (predominantly BA 23/24). Smaller regions of differentially lower activation in the ALS group were detected in the left precentral gyrus (BA 4/6) and left medial frontal gyrus (BA 6). Lower levels of differential activation were found in clusters involving the brainstem and the lingual and fusiform gyrus. Differences in cerebellar activation were also observed (Fig. 2). increased activation in the ALS group relative to healthy controls. No areas of decreased activation in the ALS group relative to controls were observed

\section{Discussion}

Our findings demonstrate that, despite not achieving statistically significant between-group differences in behavioural measures during fMRI scanning, the ALS group showed altered patterns of brain activation in comparisons designed to elicit the Stroop effect and also the NP effect, when compared to healthy controls.

\section{Stroop effect}

Both groups' RT data indicated that they demonstrated the presence of a Stroop effect. Although the RT differences and numbers of errors made during the control and Stroop tasks failed to detect statistically significant differences between the ALS and control groups, we nonetheless found a large effect size $(d=0.809)$ for the Stroop effect. The direction of this difference suggested that the ALS group was more susceptible to the interference between the 
Table 3 Regions of significant difference (at $p=0.001$ ) in activation for the Stroop effect (i.e. Stroop minus control condition) between ALS patients and healthy controls

\begin{tabular}{|c|c|c|c|c|c|c|}
\hline \multirow[t]{2}{*}{ Cerebral region } & \multirow[t]{2}{*}{ Brodmann area(s) } & \multirow[t]{2}{*}{ Side } & \multicolumn{3}{|c|}{ Coordinates of centroid point } & \multirow[t]{2}{*}{ Area (voxels) } \\
\hline & & & $X$ & $\mathrm{Y}$ & $\mathrm{Z}$ & \\
\hline \multicolumn{7}{|l|}{$A L S>C O N$} \\
\hline \multirow[t]{2}{*}{ Hippocampus } & - & $\mathrm{L}$ & -29 & -9 & -20 & 13 \\
\hline & & $\mathrm{L}$ & -39 & -19 & -16 & 124 \\
\hline \multirow[t]{2}{*}{ Inferior temporal gyrus } & 20,37 & $\mathrm{~L}$ & -51 & -14 & -20 & 17 \\
\hline & & $\mathrm{L}$ & -60 & -49 & -12 & 7 \\
\hline \multirow[t]{3}{*}{ Parahippocampal gyrus } & $19,28,36$ & $\mathrm{~L}$ & -34 & -26 & -20 & 30 \\
\hline & & $\mathrm{L}$ & -40 & -43 & -8 & 26 \\
\hline & & $\mathrm{L}$ & -38 & -44 & -4 & 4 \\
\hline \multirow[t]{3}{*}{ Fusiform gyrus } & 18,37 & $\mathrm{~L}$ & -43 & -52 & -16 & 8 \\
\hline & & $\mathrm{L}$ & -8 & -85 & -16 & 117 \\
\hline & & $\mathrm{L}$ & -27 & -96 & -16 & 20 \\
\hline \multirow[t]{6}{*}{ Middle temporal gyrus } & $20,21,39$ & $\mathrm{~L}$ & -49 & -28 & -12 & 98 \\
\hline & & $\mathrm{L}$ & -55 & -33 & -8 & 110 \\
\hline & & $\mathrm{L}$ & -51 & -21 & -4 & 7 \\
\hline & & $\mathrm{L}$ & -58 & -30 & -4 & 13 \\
\hline & & $\mathrm{L}$ & -53 & -47 & 8 & 20 \\
\hline & & $\mathrm{L}$ & -40 & -67 & 20 & 71 \\
\hline \multirow[t]{3}{*}{ Lingual gyrus } & 18 & $\mathrm{~L}$ & -16 & -84 & -8 & 116 \\
\hline & & $\mathrm{L}$ & -11 & -74 & -8 & 14 \\
\hline & & $\mathrm{L}$ & -14 & -89 & -8 & 56 \\
\hline \multirow[t]{6}{*}{ Superior temporal gyrus } & $21,22,42$ & $\mathrm{~L}$ & -51 & -20 & 1 & 10 \\
\hline & & $\mathrm{L}$ & -51 & -20 & 4 & 10 \\
\hline & & $\mathrm{L}$ & -56 & -44 & 12 & 19 \\
\hline & & $\mathrm{L}$ & -47 & -53 & 12 & 25 \\
\hline & & $\mathrm{L}$ & -48 & -47 & 16 & 219 \\
\hline & & $\mathrm{L}$ & -49 & -36 & 20 & 124 \\
\hline \multirow[t]{10}{*}{ Cingulate gyrus } & 24,32 & $\mathrm{~L}$ & -3 & 35 & 12 & 6 \\
\hline & & $\mathrm{L}$ & -12 & 42 & 16 & 4 \\
\hline & & $\mathrm{L}$ & -12 & 34 & 20 & 15 \\
\hline & & $\mathrm{L}$ & -22 & 24 & 20 & 15 \\
\hline & & $\mathrm{L}$ & -9 & 36 & 24 & 24 \\
\hline & & $\mathrm{L}$ & -23 & 23 & 24 & 4 \\
\hline & & $\mathrm{L}$ & -8 & 11 & 28 & 13 \\
\hline & & $\mathrm{L}$ & -10 & 26 & 32 & 117 \\
\hline & 24,32 & $\mathrm{R}$ & 1 & 34 & 16 & 5 \\
\hline & & $\mathrm{R}$ & 2 & 35 & 20 & 4 \\
\hline Insula & - & $\mathrm{L}$ & -35 & 24 & 12 & 37 \\
\hline Trans temporal gyrus & 41 & $\mathrm{~L}$ & -50 & -19 & 12 & 13 \\
\hline Middle occipital gyrus & 19 & $\mathrm{~L}$ & -45 & -71 & 12 & 8 \\
\hline Caudate nucleus & - & $\mathrm{L}$ & -25 & 26 & 16 & 109 \\
\hline \multirow[t]{4}{*}{ Medial frontal gyrus } & 9 & $\mathrm{R}$ & 3 & 38 & 24 & 3 \\
\hline & & $\mathrm{R}$ & 3 & 38 & 28 & 4 \\
\hline & 9,32 & $\mathrm{~L}$ & -9 & 35 & 28 & 33 \\
\hline & & $\mathrm{L}$ & -10 & 25 & 35 & 111 \\
\hline Postcentral gyrus & 2 & $\mathrm{~L}$ & -57 & -16 & 24 & 3 \\
\hline
\end{tabular}


Table 3 continued

\begin{tabular}{|c|c|c|c|c|c|c|}
\hline \multirow[t]{2}{*}{ Cerebral region } & \multirow[t]{2}{*}{ Brodmann area(s) } & \multirow[t]{2}{*}{ Side } & \multicolumn{3}{|c|}{ Coordinates of centroid point } & \multirow[t]{2}{*}{ Area (voxels) } \\
\hline & & & $X$ & $\mathrm{Y}$ & $\mathrm{Z}$ & \\
\hline \multirow[t]{5}{*}{ Inferior parietal } & \multirow[t]{5}{*}{39,40} & $\mathrm{~L}$ & -48 & -43 & 24 & 56 \\
\hline & & $\mathrm{L}$ & -48 & -62 & 24 & 7 \\
\hline & & $\mathrm{L}$ & -41 & -40 & 40 & 73 \\
\hline & & $\mathrm{L}$ & -49 & -36 & 45 & 26 \\
\hline & & $\mathrm{L}$ & -35 & -44 & 45 & 19 \\
\hline \multirow[t]{3}{*}{ Superior occipital gyrus } & \multirow[t]{3}{*}{19} & $\mathrm{~L}$ & -36 & -73 & 24 & 60 \\
\hline & & $\mathrm{L}$ & -37 & -73 & 28 & 59 \\
\hline & & $\mathrm{L}$ & -38 & -72 & 32 & 22 \\
\hline \multirow[t]{5}{*}{ Supramarginal gyrus } & \multirow[t]{5}{*}{40} & $\mathrm{~L}$ & -53 & -38 & 28 & 23 \\
\hline & & $\mathrm{L}$ & -38 & -46 & 28 & 13 \\
\hline & & $\mathrm{L}$ & -55 & -39 & 32 & 3 \\
\hline & & $\mathrm{L}$ & -35 & -43 & 32 & 14 \\
\hline & & $\mathrm{L}$ & -38 & -41 & 35 & 63 \\
\hline
\end{tabular}

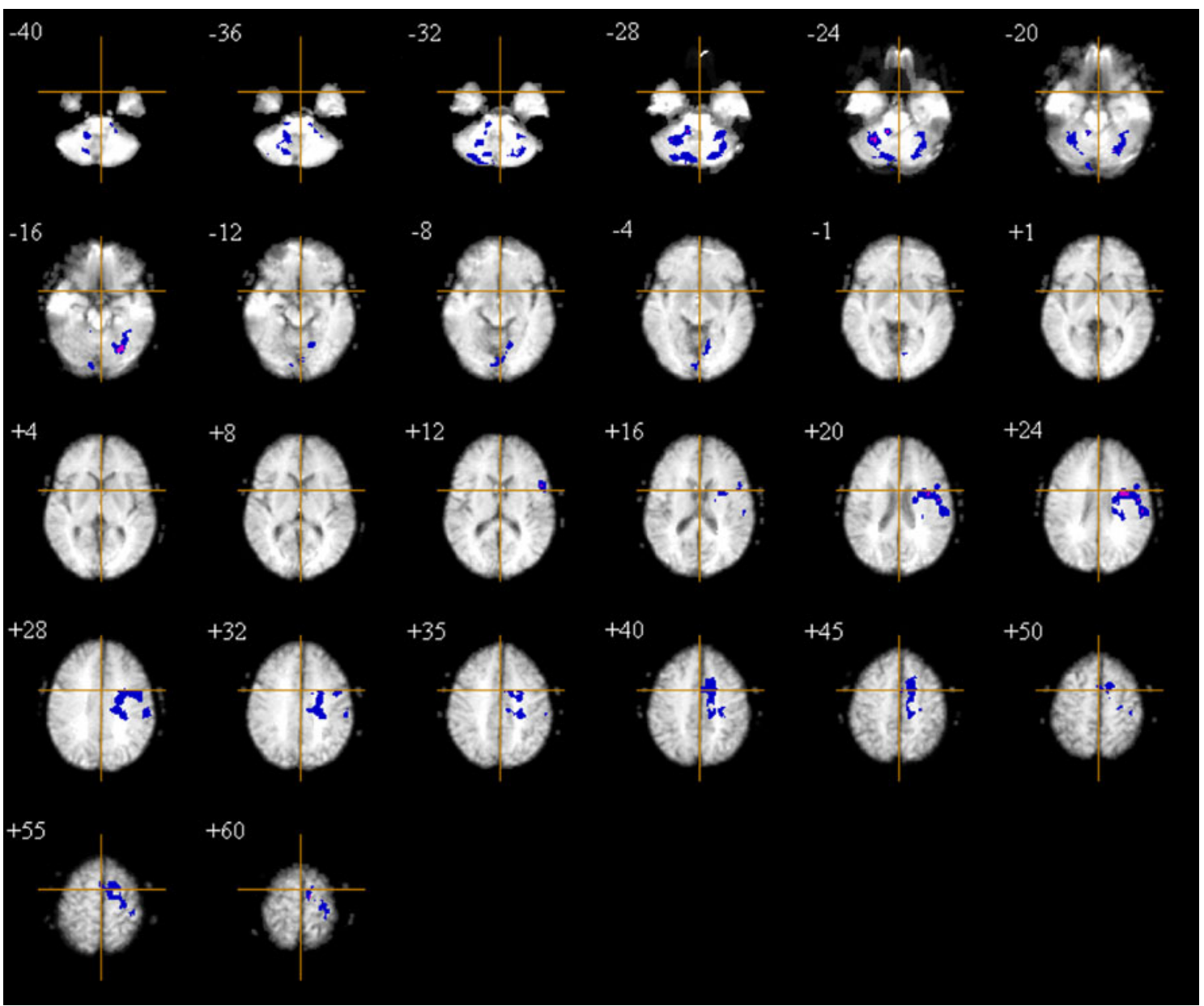

Fig. 2 Brain regions differentially activated in the ALS and healthy control groups during the negative priming minus Stroop conditions (negative priming effect) at $p=0.001$. All regions demonstrating significant between-group activation (shown in blue) showed decreased activation in the ALS group relative to healthy controls. No areas of increased activation in the ALS group relative to controls were observed 
Table 4 Regions of significant difference (at $p=0.001$ ) in activation for the negative priming effect (i.e. negative priming minus Stroop condition) between ALS patients and healthy controls

\begin{tabular}{|c|c|c|c|c|c|c|}
\hline \multirow[t]{2}{*}{ Cerebral region } & \multirow[t]{2}{*}{ Brodmann area(s) } & \multirow[t]{2}{*}{ Side } & \multicolumn{3}{|c|}{ Coordinates of centroid point } & \multirow[t]{2}{*}{ Area (voxels } \\
\hline & & & $X$ & $\mathrm{Y}$ & $\mathrm{Z}$ & \\
\hline \multicolumn{7}{|l|}{$C O N>A L S$} \\
\hline \multirow[t]{4}{*}{ Brain stem } & - & $\mathrm{L}$ & -12 & -39 & -40 & 4 \\
\hline & & $\mathrm{L}$ & -12 & -39 & -36 & 5 \\
\hline & & $\mathrm{R}$ & 17 & -38 & -36 & 8 \\
\hline & & $\mathrm{R}$ & 14 & -39 & -32 & 13 \\
\hline Fusiform gyrus & 18 & $\mathrm{R}$ & 12 & -90 & -16 & 78 \\
\hline \multirow[t]{3}{*}{ Lingual gyrus } & 17,18 & $\mathrm{R}$ & 11 & -89 & -12 & 4 \\
\hline & & $\mathrm{R}$ & 2 & -85 & -8 & 35 \\
\hline & & $\mathrm{L}$ & -6 & -75 & -1 & 5 \\
\hline Cuneus & 17 & $\mathrm{R}$ & 5 & -89 & -4 & 17 \\
\hline \multirow[t]{9}{*}{ Precentral gyrus } & 4,6 & $\mathrm{~L}$ & -51 & 5 & 12 & 33 \\
\hline & & $\mathrm{L}$ & -48 & 5 & 16 & 12 \\
\hline & & $\mathrm{L}$ & -40 & -11 & 20 & 164 \\
\hline & & $\mathrm{L}$ & -44 & -4 & 32 & 16 \\
\hline & & $\mathrm{L}$ & -25 & -23 & 45 & 4 \\
\hline & & $\mathrm{L}$ & -26 & -21 & 50 & 7 \\
\hline & & $\mathrm{L}$ & -39 & -27 & 50 & 6 \\
\hline & & $\mathrm{L}$ & -37 & -28 & 55 & 10 \\
\hline & & $\mathrm{L}$ & -28 & -23 & 60 & 38 \\
\hline Putamen & - & $\mathrm{L}$ & -27 & -4 & 16 & 20 \\
\hline Superior temporal gyrus & 42 & $\mathrm{~L}$ & -53 & -27 & 16 & 6 \\
\hline \multirow[t]{11}{*}{ Cingulate gyrus } & $23,24,29$ & $\mathrm{~L}$ & -28 & -32 & 20 & 3 \\
\hline & 31 & $\mathrm{~L}$ & -24 & -28 & 24 & 33 \\
\hline & & $\mathrm{L}$ & -29 & -14 & 28 & 174 \\
\hline & & $\mathrm{L}$ & -21 & -19 & 32 & 107 \\
\hline & & $\mathrm{L}$ & -20 & -6 & 35 & 43 \\
\hline & & $\mathrm{L}$ & -21 & -28 & 35 & 42 \\
\hline & & $\mathrm{L}$ & -12 & 0 & 40 & 116 \\
\hline & & $\mathrm{L}$ & -26 & -26 & 40 & 21 \\
\hline & & $\mathrm{L}$ & -14 & -28 & 40 & 27 \\
\hline & & $\mathrm{L}$ & -14 & -6 & 45 & 113 \\
\hline & & $\mathrm{L}$ & -3 & 0 & 45 & 8 \\
\hline Postcentral gyrus & 3 & $\mathrm{~L}$ & -40 & -11 & 24 & 177 \\
\hline \multirow[t]{2}{*}{ Inferior parietal } & 40 & $\mathrm{~L}$ & -54 & -27 & 28 & 30 \\
\hline & & $\mathrm{L}$ & -55 & -28 & 35 & 3 \\
\hline Supramarginal gyrus & 40 & $\mathrm{~L}$ & -55 & -29 & 35 & 11 \\
\hline \multirow[t]{4}{*}{ Medial frontal gyrus } & 6 & $\mathrm{~L}$ & -13 & 4 & 50 & 30 \\
\hline & & $\mathrm{L}$ & -1 & 3 & 50 & 14 \\
\hline & & $\mathrm{L}$ & -15 & -4 & 55 & 122 \\
\hline & & $\mathrm{L}$ & -10 & -5 & 60 & 23 \\
\hline
\end{tabular}

colour words and inks, but that our findings were likely to have been affected by the small sample size.

The ALS group showed increased activation in regions associated with word representation (left superior temporal gyrus; BA 22) and spatial attention (left inferior parietal cortex BA 39/40). The latter differences may represent covert shifts in attention between stimuli and response requirements [26]. In addition, the large region of relatively increased activation in the anterior cingulate (BA 24/32) is not dissimilar from a cluster of relatively decreased 
activation observed in healthy control participants [31]. Steel et al.'s [26] main area of activation when comparing the Stroop and control conditions was the cingulate gyrus, and the current findings lend support to the validity of the current scanning paradigm, as does the involvement of other regions also previously found to be implicated in the Stroop effect [26]. Previous findings in healthy controls of parrahippocampal gyrus and cerebellar activation [26] during the Stroop effect mirror areas differentiating between the ALS and control groups in the current study, although between-group differences in hippocampal activation were also seen in the present study. Whether this reflects greater difficulty on the part of the ALS in remembering on a trial-by-trial basis to name the ink rather than reading the word cannot be determined from the response data, but may be one factor underlying the somewhat greater Stroop effect for the ALS group compared to controls. Cerebellar activation during the Stroop task has been reported in other patient groups (e.g. [49]). As with their findings [49], the increased activation of the cerebellum during the Stroop effect comparison may reflect the maintained difficulty of the task for the ALS group such that the task failed to become automatic for them. The RT findings suggest that the ALS patients found the inhibitory task more difficult than controls, resulting in greater activation of implicated brain regions. Further substantial regions of increased cortical activation were observed in the left middle temporal gyrus (BA 20/21) and the left fusiform and lingual gyri (BA 17, 18; reported also by Steel et al. [26]). Both the middle temporal gyrus [50] and the lingual-fusiform border [32] have been implicated in the processing of words or non-words rather than strings of consonants. Thus the areas of increased activation might suggest that the ALS patients had greater difficulty in suppressing word reading in order to name the colour inks.

\section{Negative priming effect}

Behavioural data during scanning did not unequivocally support our previous finding [6] that the ALS patients might show reduced inhibitory processing in the comparison between the NP and Stroop tests. Although there was a tendency for the ALS group to make more errors than healthy controls during the NP trials, neither group showed statistically different RTs in the comparison between the NP and Stroop conditions. However both groups did show their longest mean RTs in the NP condition, providing some validity for the paradigm. The reason for the failure to demonstrate a statistically significant NP effect (in terms of RT measures) in either group is unclear, but both groups' RTs showed considerable variability in the extent of their NP effect (Table 2). Our sample was older than those reported in other imaging studies of NP [26, 30], and varying findings have been reported concerning whether increasing age reduces the magnitude of the NP effect [51]. In addition the need for a long response time, the periodic block scanning design and the resulting short blocks of NP trials may have provided insufficient opportunity for the NP trials to have become sufficiently differentiated from the Stroop trials in terms of their processing demands. Nonetheless, our imaging findings suggest differential patterns of stimulus processing by the two groups within the NP effect scanning comparison. However, our following interpretation of these findings can only be speculative at this stage, given our small sample size.

In healthy controls, greater activation of specific brain regions during NP trials relative to Stroop trials has been reported [26], indicating the additional cognitive load imposed by responding to a previously cognitively inhibited stimulus. In the current study, relatively large clusters reflecting reduced cerebral activation in the ALS group compared to controls were found in two areas previously associated with performance on this task: the left precentral gyrus (BA 4/6) and left medial frontal gyrus (BA 6). It has been suggested [26] that the precentral gyrus may contribute to the processes of articulation of the target colour at a specific trial; reduced activation in this region in the ALS group might reflect higher levels of residual activation of this target as a result of poorer suppression of the same word when it appeared as a distractor in the previous trial. The medial frontal cortex is likely to contribute to the working memory or executive demands of the task (e.g. internal representations of previous stimuli, self monitoring of responses and the inhibition of unwanted responses). The relatively reduced activation in this region in the ALS group may reflect less effective cognitive inhibition of the colour name in previous trials and therefore a reduced demand for executive processes on current trials. Relatively reduced cerebral activation in the left anterior and posterior cingulate gyrus (BA 23/24) in the ALS group was not consistent with findings in studies of NP in healthy controls [26] where no differential activation of these areas was reported. However, the reduced activation of these regions in the ALS patients suggests that the cognitive response inhibition and attentional demands of this test may be processed differently by the ALS and control participants. It also suggests that reactivation of the previously suppressed stimulus is less difficult for the ALS group, due to previously less efficient response inhibition processes. While clear involvement of medial temporal regions in the NP effect has been reported [26], this was not a substantial region showing differential activation between the ALS and control groups, suggesting that the ALS group did not differ substantially from controls in their processing of the episodic retrieval component of the NP task. Overall, the finding of relatively lower activation 
in the ALS group in the prefrontal regions associated with the working memory and articulatory elements of NP performance suggests that reactivation of the previously suppressed stimulus was less difficult for the ALS group. The finding of bilateral cerebellar activation, here found to be reduced in the ALS group, was not consistent with earlier studies in controls [26]. The finding of predominantly left-sided differences between the Control and ALS groups' pattern of activation would support findings of left frontal lobe involvement in the active inhibitory processes that contribute to NP [52].

\section{Limitations of the present study}

Although a strength of the present study was the collection of online behavioural measures, the sample sizes for our groups restricted the power available to detect betweengroup differences on our behavioural measures. Power was further reduced by the failure to record behavioural measures for two ALS participants. Our study is therefore very much a pilot that needs future replication in a larger cohort. Despite the collection of measures of premorbid IQ and mood, further clarification of the extent of cognitive impairment in the ALS group from neuropsychological assessment was not available. The identification of more generalised executive dysfunction and its relationship to imaging findings in the present samples would have been informative, especially in light of the heterogeneity of cognitive involvement in ALS [3, 53]. In addition, our scanning study design required the exclusion of ALS patients with very marked speech impairments (i.e. more severe bulbar impairment), and since this may have excluded patients with more pronounced cognitive impairment [6], this may have influenced our findings.

Within our imaging paradigm, the sparse sequence design and the need to accommodate for patients' dysarthria resulted in longer ISIs (verbal response time) than have been used in other studies (e.g. 2,600 ms used here as compared to $1,900 \mathrm{~ms}$ elsewhere [26]). Although this will not have influenced between-condition comparisons, this does limit comparability with other studies of Stroop and NP effects, and may explain some of the observed differences in regional activation between this and other studies. In particular, it has been suggested that longer ISIs in the Stroop task lead to more 'routine' performance of the task, and it has been argued that attentional demands are reduced under such conditions, leading to reduced prefrontal activation [54]. In addition different cortical circuits may be activated at different stages of the Stroop response, with frontocentral regions being implicated earlier in the process than temporo-parietal regions [55], and our design may have led to less frontal activation being detected. Future studies might also usefully consider the relationship between alterations in cortical inhibitory processes in ALS (e.g. $[56,57])$ and performance on measures of cognitive inhibition.

These limitations notwithstanding, our findings demonstrate the presence of greater cerebral activation in the ALS group in the Stroop effect, reflecting apparently greater difficulty in achieving response suppression. Findings of areas of relatively lower cerebral activation in the ALS group in the NP effect might support a model of stimulus processing in the ALS group where lower levels of cognitive inhibitory processes are employed, despite an absence of an unequivocal between-group difference in behavioural measures of the NP effect. These findings extend the neuroimaging correlates of cognitive impairment in patients with ALS [58] and suggest, as in other studies [8], that functional imaging may detect evidence of altered cerebral processing in ALS in the absence of marked apparent cognitive dysfunction.

Acknowledgments We confirm that we have read the journal's position on issues involved in ethical publication and affirm that this report is consistent with those guidelines. I N-D and VB were in receipt of funding from the Medical Research Council, UK. The King's MND Care \& Research Centre receives funding from the Motor Neurone Disease Association UK. AS and PNL were in receipt of funding from the Biomedical Research Centre for Mental Health at South London and Maudsley NHS Foundation Trust and King's College London, Institute of Psychiatry.

Conflict of interest The authors have no conflict of interest to declare.

Open Access This article is distributed under the terms of the Creative Commons Attribution Noncommercial License which permits any noncommercial use, distribution, and reproduction in any medium, provided the original author(s) and source are credited.

\section{References}

1. Phukan J, Pender NP, Hardiman O (2007) Cognitive impairment in amyotrophic lateral sclerosis. Lancet Neurology 6:994-1003

2. Raaphorst J, De Visser M, Linssen WHJP, De Haan RJ, Schmand B (2010) The cognitive profile of amyotrophic lateral sclerosis: a meta-analysis. Amyotroph Lateral Scler 11:27-37

3. Ringholz GM, Appel SH, Bradshaw M, Cooke NA, Mosnik DM, Schulz PE (2005) Prevalence and patterns of cognitive impairment in sporadic ALS. Neurology 65:586-590

4. Strong MJ (2008) The syndromes of frontotemporal dysfunction in amyotrophic lateral sclerosis. Amyotroph Lateral Scler 9:323-338

5. Goldstein LH (2006) Control of symptoms: cognitive dysfunction. In: Oliver D, Borasio GD, Walsh D (eds) Palliative care in amyotrophic lateral sclerosis. Oxford University Press, Oxford, pp 111-127

6. Abrahams S, Goldstein LH, Al Chalabi A et al (1997) Relation between cognitive dysfunction and pseudobulbar palsy in amyotrophic lateral sclerosis. J Neurol Neurosurg Psychiatry 62:464-472 
7. Abrahams S, Goldstein LH, Lloyd CM, Brooks DJ, Leigh PN (1995) Cognitive deficits in non-demented amyotrophic lateral sclerosis patients: a neuropsychological investigation. J Neurol Sci 129(Suppl):54-55

8. Abrahams S, Goldstein LH, Simmons A et al (2004) Word retrieval in amyotrophic lateral sclerosis: a functional magnetic resonance imaging study. Brain 127:1507-1517

9. Abrahams S, Leigh PN, Goldstein LH (2005) Cognitive change in ALS: a prospective study. Neurology 64:1222-1226

10. Abrahams S, Leigh PN, Harvey A, Vythelingum GN, Grise D, Goldstein LH (2000) Verbal fluency and executive dysfunction in amyotrophic lateral sclerosis (ALS). Neuropsychologia 38:734-747

11. Kew JJ, Goldstein LH, Leigh PN et al (1993) The relationship between abnormalities of cognitive function and cerebral activation in amyotrophic lateral sclerosis. A neuropsychological and positron emission tomography study. Brain 116:1399-1423

12. Lomen-Hoerth C, Murphy J, Langmore S, Kramer JH, Olney RK, Miller B (2003) Are amyotrophic lateral sclerosis patients cognitively normal? Neurology 60:1094-1097

13. Flaherty-Craig C, Brothers A, Dearman B, Eslinger P, Simmons Z (2009) Penn state screen exam for the detection of frontal and temporal dysfunction syndromes: application to ALS. Amyotroph Lateral Scler 10:107-112

14. Abrahams S, Goldstein LH, Kew JJ, Brooks DJ, Lloyd CM, Frith CD, Leigh PN (1996) Frontal lobe dysfunction in amyotrophic lateral sclerosis. A PET study. Brain 119:2105-2120

15. Abrahams S, Leigh PN, Kew JJ, Goldstein LH, Lloyd CM, Brooks DJ (1995) A positron emission tomography study of frontal lobe function (verbal fluency) in amyotrophic lateral sclerosis. J Neurol Sci 129(Suppl):44-46

16. Wicks P, Turner MR, Abrahams S, Hammers A, Brooks DJ, Leigh PN, Goldstein LH (2008) Neuronal loss associated with cognitive performance in amyotrophic lateral sclerosis: an $\left({ }^{11} \mathrm{C}\right)$ flumazenil PET study. Amyotroph Lateral Scler 9:43-49

17. Abrahams S, Goldstein LH, Suckling J et al (2005) Frontotemporal white matter changes in amyotrophic lateral sclerosis. J Neurology 253:321-331

18. Lule D, Ludolph AC, Kassubek J (2009) MRI-based functional neuroimaging in ALS: an update. Amyotrophic Lateral Sclerosis $10: 258-268$

19. Chari G, Shaw PJ, Sahgal A (1996) Nonverbal visual attention, but not recognition memory or learning processes are impaired in motor neurone disease. Neuropsychologia 34:377-385

20. Frank B, Haas J, Heinze HJ, Stark E, Munte TF (1997) Relation of neuropsychological and magnetic resonance findings in amyotrophic lateral sclerosis: evidence for subgroups. Clin Neurol Neurosurg 99:79-86

21. Hanagasi HA, Gurvit IH, Ermutlu N et al (2002) Cognitive impairment in amyotrophic lateral sclerosis: evidence from neuropsychological investigation and event-related potentials. Cogn Brain Res 14:234-244

22. Ludolph AC, Langen KJ, Regard M et al (1992) Frontal lobe function in amyotrophic lateral sclerosis: a neuropsychologic and positron emission tomography study. Acta Neurol Scand 85:81-89

23. Pinkhardt EH, Jurgens R, Becker W et al (2008) Signs of impaired selective attention in patients with amyotrophic lateral sclerosis. J Neurol 255:532-538

24. Stroop J (1935) Studies of interference in serial verbal reactions. Q J Exp Psychol 18:643-661

25. Tipper SP, Cranston M (1985) Selective attention and priming: inhibitory and facilitatory effects of ignored primes. Q J Exp Psychol A Hum Exp Psychol 37:591-611

26. Steel C, Haworth EJ, Peters E et al (2001) Neuroimaging correlates of negative priming. NeuroReport 12:3619-3624
27. Egner T, Hirsch $\mathbf{J}$ (2005) Where memory meets attention: neural substrates of negative priming. J Cogn Neurosci 17:1774-1784

28. Metzler C, Parkin AJ (2000) Reversed negative priming following frontal lobe lesions. Neuropsychologia 38:363-379

29. Peters ER, Pickering AD, Hemsley DR (1994) 'Cognitive inhibition' and positive symptomatology in schizotypy. Br J Clin Psychol 33:33-48

30. Ungar L, Nestor PG, Niznikiewicz MA, Wible CG, Kubicki M (2010) Color Stroop and negative priming in schizophrenia: an fMRI study. Psychiat Res Neuroimaging 181:24-29

31. Leung H-C, Skudlarski P, Gatenby JC, Peterson BS, Gore JC (2000) An event-related functional MRI study of the Stroop color word interference task. Cereb Cortex 10:552-560

32. Peterson BS, Skudlarski P, Gatenby JC, Zhang H, Anderson AW, Gore JC (1999) An fMRI study of Stroop word-color interference: evidence for cingulate subregions subserving multiple distributed attentional systems. Biol Psychiatry 45:1237-1258

33. Maekawa S, Al Sarraj S, Kibble M et al (2004) Cortical selective vulnerability in motor neuron disease: a morphometric study. Brain 127:1237-1251

34. Stanton BR, Williams VC, Leigh PN et al (2007) Altered cortical activation during a motor task in ALS: evidence for involvement of central pathways. J Neurol 254:1260-1267

35. Brooks BR, Miller RG, Swash M, Munsat TL, World Federation of Neurology Research Group on Motor Neuron Diseases (2000) El Escorial revisited: revised criteria for the diagnosis of amyotrophic lateral sclerosis. Amyotroph Lateral Scler Other Motor Neuron Dis 1:293-299

36. Mustfa N, Walsh E, Bryant V et al (2006) The effect of noninvasive ventilation on ALS patients and their caregivers. Neurology 66:1211-1217

37. Newsom-Davis IC, Lyall RA, Leigh PN, Moxham J, Goldstein LH (2001) The effect of non-invasive positive pressure ventilation (NIPPV) on cognitive function in amyotrophic lateral sclerosis (ALS): a prospective study. J Neurol Neurosurg Psychiatry 71:482-487

38. Hillel AD, Miller RM, Yorkston K, McDonald E, Norris FH, Konikow N (1989) Amyotrophic lateral sclerosis severity scale. Neuroepidemiology 8:142-150

39. Nelson HE, Willison J (1991) National Adult Reading Test (NART) second edition. NFER-Nelson, Windsor

40. Zigmond AS, Snaith RP (1983) The hospital anxiety and depression scale. Acta Psychiat Scand 67:361-370

41. Simmons A, Moore E, Williams SCR (1999) Quality control for functional magnetic resonance imaging using automated data analysis and Shewhart charting. Magn Res Med 41:1274-1278

42. Bullmore ET, Brammer MJ, Rabe-Hesketh S et al (1999) Methods for diagnosis and treatment of stimulus-correlated motion in generic brain activation studies using fMRI. Hum Brain Map 7:38-48

43. Bullmore E, Brammer M, Williams SCR et al (1996) Statistical methods of estimation and inference for functional MR image analysis. Magn Reson Med 35:261-277

44. Bullmore ET, Long C, Suckling J et al (2001) Colored noise and computational inference in neurophysiological (fMRI) time series analysis: resampling methods in time and wavelet domains. Hum Brain Mapp 12:61-78

45. Brammer MJ, Bullmore ET, Simmons A et al (1997) Generic brain activation mapping in functional magnetic resonance imaging: a nonparametric approach. Magn Res Imaging 15:763-770

46. Talairach J, Tournoux P (1988) CoPlanar sterotaxic atlas of the human brain. Thieme, Stuttgart

47. Bullmore ET, Suckling J, Overmeyer S, Rabe-Hesketh S, Taylor E, Brammer MJ (1999) Global, voxel, and cluster tests, by theory 
and permutation, for a difference between two groups of structural MR images of the brain. IEEE Transact Med Imag 18:32-42

48. Cohen JA (1992) A power primer. Psychol Bull 112:155-159

49. Rocca MA, Valsasina P, Ceccarelli A et al (2009) Structural and functional MRI correlates of Stroop control in benign MS. Hum Brain Mapp 30:276-290

50. Price CJ, Wise RJS, Watson JDG, Patterson K, Howard D, Frackowiak RSJ (1994) Brain activity during reading. The effects of exposure duration and task. Brain 117:1255-1269

51. Gamboz N, Russo R, Fox E (2002) Age differences and the identity negative priming effect: an updated meta-analysis. Psychol Aging 17:525-531

52. McDonald CR, Bauer RM, Filoteo JV, Grande L, Roper SN, Gilmore R (2005) Attentional inhibition in patients with focal frontal lobe lesions. J Clin Exp Neuropsychol 27:485-503

53. Wicks P, Abrahams S, Papps B, Leigh PN, Goldstein LH (2009) SOD1 and cognitive dysfunction in familial amyotrophic lateral sclerosis. J Neurol 256:234-241
54. Bench C, Frith CD, Grasby PM et al (1993) Investigations of the functional anatomy of attention using the Stroop test. Neuropsychologia 31:907-922

55. Xiao X, Qiu J, Zhang Q (2009) The dissociation of neural circuits in a Stroop task. NeuroReport 20:674-678

56. Turner MR, Osei-Lah AD, Hammers A et al (2005) Abnormal cortical excitability in sporadic but not homozygous D90A SOD1 ALS. J Neurol Neurosurg Psychiatry 76:1279-1285

57. Steve V, Kiernan MC (2009) Abnormalities of intracortical pathways in amyotrophic lateral sclerosis-developing new therapeutic targets. J Clin Neurosci Conf Proc 1516-1517

58. Tsermentseli S, Goldstein LH, Leigh PN (2011) The anatomy of cognitive impairment in amyotrophic lateral sclerosis: more than frontal lobe dysfunction. Cortex. doi:10.1016/j/cortex.2011. 02.004 